\section{Clarification of OLAW's position on rodent cage changes}

\section{To the editor:}

The April 2013 issue of Lab Animal included an article written by Gregory P. Boivin, DVM, MS, DACLAM, titled "Availability of feces-free areas in rodent shoebox cages"1. In the article, Dr. Boivin describes a study documenting the presence of urine and feces at specific time points in shoebox cages housing rodents in varying densities. He concludes that the recommendation of the eighth edition of the Guide for the Care and Use of Laboratory Animals ${ }^{2}$ (the Guide) to provide terrestrial mammals with sufficient space free of urine and feces in which to rest is not in the best interest of the rodents and that to meet this guidance would require daily cage changes in some cases.

The Office of Laboratory Animal Welfare (OLAW) at the National Institutes of Health would like to clarify OLAW's position and interpretation of the Guide on rodent cage changes. As Dr. Boivin points out, on page 56, the Guide states, “...there must be sufficient space to comfortably rest away from areas soiled by urine and feces" 2 . However, this statement is taken from the section titled "Space" and the subsection "General Considerations for All Animals." As such, it addresses the considerations that are important in deciding the pen or cage size of research animal subjects.
The Guide addresses cage-change husbandry concerns in a section on page 70 titled "Bedding/Substrate Change" 2 . In this section, the Guide states, "Soiled bedding should be removed and replaced with fresh materials as often as necessary to keep the animals clean and dry and to keep pollutants, such as ammonia, at a concentration below levels irritating to mucous membranes. The frequency of bedding change depends on multiple factors, such as species, number, and size of the animal... urinary and fecal output and the appearance and wetness of bedding... There is no absolute minimal frequency of bedding changes; the choice is a matter of professional judgment and consultation between the investigator and animal care personnel" 2 .

OLAW considers both statements in the Guide to be accurate and in the best interests of the animal and the research, when correctly applied.

\section{Susan Brust Silk, MS \& Patricia Brown, VMD, MS, DACLAM}

Office of Laboratory Animal Welfare, Office of Extramural Research, National Institutes of Health, US Department of Health and Human Services, Bethesda, MD. e-mail:olaw@od.nih.gov

1. Boivin, G.P. Availability of feces-free areas in rodent shoebox cages. Lab Anim. (NY) 42, 135-141 (2013).

2. Institute for Laboratory Animal Research. Guide for the Care and Use of Laboratory Animals 8th edn. 56, 70 (National Academies Press, Washington, DC, 2011). 